Die wissenschaftspolitischen Empfehlungen des WBGU

\section{Die "große Transformation“ braucht Bewegung in der Wissenschaft}

Mitte Juni hat der Wissenschaftliche Beirat Globale Umweltveränderungen (WBGU) sein neues Hauptgutachten zur „Großen Transformation"vorgelegt. Eine besondere Brisanz liegt in den wissenschaftspolitischen Empfehlungen. Was bedeuten diese Empfehlungen für die Wirtschafts- und Managementwissenschaften? Von Uwe Schneidewind

$\mathbf{S}^{\mathrm{n}}$ it Mitte Juni liegt die vollständige Fassung des Hauptgutachtens des Wissenschaftlichen Beirats für Globale Umweltveränderungen (WBGU) der deutschen Bundesregierung vor (WBGU 2011). Unter dem Titel „Welt im Wandel - Gesellschaftsvertrag für eine Große Transformation" zeigt der WBGU auf, dass die Transformation zu einer globalen Nachhaltigen Gesellschaft weit mehr ist als eine technologische Herausforderung. Sie stellt vielmehr einen umfassenden sozialen und kulturellen Wandel dar, der einen neuen Gesellschaftsvertrag braucht.

\section{Neuer Vertrag zwischen Wis- senschaft und Gesellschaft}

Ein wichtiger Teil dieses neuen Gesellschaftsvertrages muss aus Sicht des

Abbildung 1: Das „transformative Quartett der Wissensgesellschaft"

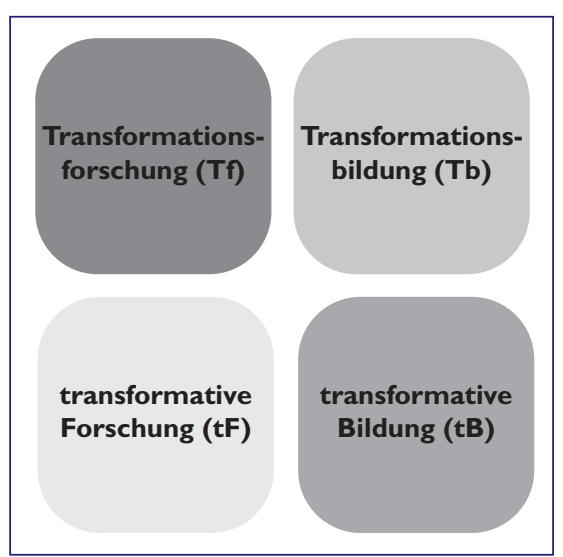

Quelle: WBGU 2011
WBGU ein veränderter „Vertrag zwischen Wissenschaft und Gesellschaft" (S. 342) sein. Auf über 40 Seiten untersucht das Gutachten die aktuelle Forschungspolitik auf europäischer und nationaler Ebene und leitet umfassende Forderungen an eine künftige Wissenschaftspolitik ab. Eine solch prominente Einmischung in die Wissenschaftspolitik hat es bisher von keinem umwelt- und nachhaltigkeitspolitischen Beratergremium der Bundesregierung gegeben.

\section{Stärkung von trans- formativer Wissenschaft}

Orientierungspunkt für die Wissenschaftsstrategie des WBGU ist ein transformatives Quartett der Wissensgesellschaft (siehe Abbildung 1). Demnach bedarf es sowohl einer Transformationsforschung, das heißt einer Forschung, die Zusammenhänge von umfassenden gesellschaftlichen Transformationsprozessen versteht, als auch einer transformativen Forschung, das heißt einer Forschung, die transformative Prozesse aktiv befördert. In analoger Weise gilt dies für den Bildungsbereich - Forschung und Bildung sind dabei eng miteinander $\mathrm{zu}$ verzahnen.

Die Idee der Transformations- und der transformativen Forschung deckt sich weitgehend mit dem wissenschaftstheoretisch etablierten Programm einer transdisziplinären Nachhaltigkeitswissenschaft, die darauf zielt, System-, Ziel- und Transformationswissen $\mathrm{zu}$ generieren und sich konsequent an gesellschaftlichen Schlüsselfragen auszurichten.

\section{Ernüchterndes Fazit}

Die Analyse der bestehenden europäischen und nationalen Forschungspolitik im Hinblick auf einen solchen Anspruch fällt ernüchternd aus. Das Gutachten analysiert einerseits die Forschungsprogramme auf Ebene der Europäischen Union. Dazu gehören das Grünbuch 2007 des Europäischen Forschungsraumes, das Kooperationsprogramm im 7. EU-Forschungsrahmenprogramm, das Joint Programming, die Joint Technology Initiatives sowie das European Institute of Technology EIT mit seinen Knowledge Innovation Clustern (KICs). Andererseits werden auch die Programme auf Bundesebene untersucht. Dazu gehören übergreifende Programme wie die HightechStrategie der Bundesregierung, die Nationale Forschungsstrategie Bioökonomie, das Rahmenprogramm Forschung für eine Nachhaltige Entwicklung (FONA), die sozial-ökologische Forschung und den Pakt für Forschung und Innovation sowie spezifische Forschungsprogramme in den Bereichen Energie, Urbanisierung und Landnutzung.

Das Ergebnis lautet fast überall ähnlich: Klimaverträglichkeit ist häufig ein Ziel, steht aber nur nebenrangig neben ökonomischen Zielen (siehe auch das Gesamtfazit in WBGU 2011, S. 370 f.). Interdisziplinarität wird in vielen Programmen proklamiert, aber nur selten systematisch eingelöst. Fast alle Programme bleiben stark technologisch orientiert, soziale und kulturelle Innovationen spielen kaum eine Rolle. Die wenigen wirklichen transformativen Forschungsprogramme, beispielsweise in der Stadtforschung oder der sozial-ökologischen Forschung, sind im Vergleich zu anderen Programmen finanziell äußerst gering ausgestattet.

Dabei macht der WBGU deutlich, dass die Defizite weit tiefer gehen als falsche Prioritätensetzungen bei Forschungsprogrammen. Es sind vielmehr grundsätzliche strukturelle Hindernisse und „falsch ausgerichtete Anreizsysteme“ (S. 373), die 
verhindern, dass sich eine transformative Forschung bisher im Wissenschaftssystem durchsetzen kann. Damit greift das Gutachten aktuelle Analysen zu den institutionellen Defiziten für eine transdisziplinäre Nachhaltigkeitswissenschaft auf (Schneidewind 2010).

\section{Ein umfassendes Reformprogramm}

Der WBGU entwickelt in seinem Gutachten daher ein sehr umfassendes Reformprogramm (S. 377 ff.). Er plädiert unter anderem für die Einrichtung eines eigenen Forschungsfeldes „Transformationsforschung", die finanzielle Aufstockung der wenigen bisherigen transdisziplinären Forschungsprogramme wie der sozial-ökologischen Forschung (SÖF), das Nachdenken über eine Bundesuniversität, die „schwerpunktmäßig Forschung und Bildung für die Transformation zur Nachhaltigkeit betreibt", sowie eine eigene Runde der Exzellenzinitiative, die vollständig der Transformation für eine ressourcenschonende, nachhaltige und lebenswerte Gesellschaft gewidmet ist. Daneben plädiert der WBGU für transformationsrelevante Sabbaticals und die Einführung eines freiwilligen Gesellschaftsjahres „Bildung und Wissenschaft“ sowie im Bildungsbereich für die Einrichtung von „Low Carbon Business Schools“ (S. 382).

Mit diesen Empfehlungen greift der WBGU weit über Impulse für einzelne Forschungsprogramme hinaus und bewegt sich auf der Ebene der wissenschaftspolitischen Gestaltungskompetenzen von Bund und insbesondere den Ländern.

\section{Capacity Building als zentrale Herausforderung ...}

Einlösen lässt sich dieses Programm nur, wenn es $\mathrm{zu}$ einem engen $\mathrm{Zu}$ sammenspiel von Bundes- und Landespolitik und bestehenden Vorreiterinstitutionen kommt. Denn letztlich müssen die Impulse von Hochschulen und Forschungsinstituten übernommen werden, die im Zuge der gewachsenen Wissen- schaftsautonomie über hohe Freiheitsgrade verfügen.

Dabei haben viele der großen Universitäten und Forschungseinrichtungen in den letzten Jahren - unter anderem befördert durch die Exzellenzinitiative eher eine Re-Orientierung in Richtung eines klassischen Wissenschaftsverständnisses erlebt. Gerade deswegen ist es wichtig, dass in die Neu-Orientierung des Wissenschaftssystems die Pionierinstitutionen, die zum Teil seit über 20 Jahren das Programm einer transformativen Nachhaltigkeitsforschung verfolgen, einbezogen sind. Dazu gehören Institute wie das Institut für sozial-ökologische Forschung (ISOE), das Öko-Institut, das Institut für ökologische Wirtschaftsforschung (IÖW), das Institut für Energieund Umweltforschung (ifeu), das Ecologic Institut oder das Wuppertal Institut; aber auch Vorreiteruniversitäten wie die Leuphana Universität Lüneburg oder die Universität Kassel. Gerade die enge universitäre Anbindung ist für einen Kapazitätsaufbau wichtig, denn in den Universitäten entscheiden sich die Chancen für Qualifizierungspfade und akademische Karrieren im Kontext einer transformativen Wissenschaft.

Es ist daher erfreulich, dass neben den großen Forschungsgemeinschaften wie der Leibniz-Gemeinschaft, der Helmholtz-Gemeinschaft und der FraunhoferGesellschaft mit einzelnen Leitinstituten wie dem Potsdam Institut (PIK), dem Umweltforschungszentrum Leipzig (UFZ) und dem „Netzwerk Nachhaltigkeit" von 18 Fraunhofer Instituten, sich die oben genannten Vorreiter-Institute und Universitäten eng vernetzen und damit zum Motor für eine transformative Wissenschaft in Deutschland werden (Schneidewind/Lang/Ernst 2011).

\section{... gerade auch in den Wirtschaftswissenschaften}

Für die Wirtschafts- und Managementwissenschaften stellt die Orientierung zu einer transformativen Wissenschaft eine besondere Herausforderung und Chance dar. Die große Transformation ist letztlich ein gewaltiges ökonomi- sches Gestaltungsprojekt. Es entstehen neue Märkte, Geschäftsmodelle sowie neue institutionelle Rahmungen für wirtschaftliches Handeln. Die meisten einschlägigen Forschungseinrichtungen und wirtschaftswissenschaftlichen Fachbereiche der Hochschulen sind jedoch in ihren Konzepten und Modellen weiterhin konventionell aufgestellt. Die nur zaghafte Auseinandersetzung mit neuen Wachstums- und Wohlstandsmodellen ist ein Ausdruck davon. Jedoch stimmen einzelne Entwicklungen wie die Ansätze einer kulturalistischen Ökonomik (AntoniKomar/Pfriem 2009), innovative Ansätze der experimentellen Ökonomie oder auch die Nobelpreisverleihung an Elinor Ostrom und ihre wegweisenden Arbeiten zur Gemeingüternutzung optimistisch. Hier ist auf einen stärkeren transformativen Wandel zu hoffen.

\section{Literatur}

Antoni-Komar, I. / Pfriem, R.: Kulturalistische Ökonomik. Vom Nutzen einer Neuorientierung wirtschaftswissenschaftlicher Untersuchungen. Wenke2-Diskussionspapier Nr. 6/09. Oldenburg 2009.

Schneidewind, U.: Ein institutionelles Reformprogramm zur Förderung transdisziplinärer Nachhaltigkeitsforschung. In: GAIA 19, 2/2010, S.122-128.

Schneidewind, U. / Ernst, A. / Lang, D.: Institutionen für eine transformative Forschung. Zur Gründung der NaWis-Runde. In: GAIA 20, 2/2011, S. 133-135.

WBGU (Wissenschaftlicher Beirat Globale Umweltveränderungen der Bundesregierung): Welt im Wandel. Gesellschaftsvertrag für eine Große Transformation. Berlin 2011.

I AUTOR + KONTAKT

Dr. Uwe Schneidewind ist Präsident des Wuppertal Institutes und Inhaber der Professur für Innovationsmanagement und Nachhaltigkeit (Sustainable Transition Management) an der Schumpeter School der Bergischen Universität Wuppertal. Zugleich ist er Mitglied des Vorstands der VÖW.

Wuppertal Institut für Klima, Umwelt, Energie $\mathrm{GmbH}$, Döppersberg 19, 42103 Wuppertal.

E-Mail: uwe.schneidewind@wupperinst.org Weitere Beiträge und Diskussionen zum Thema auf dem Blog www.nachhaltigewissenschaft.blog.de 


\section{Lizenzhinweis}

Die Beiträge in ÖkologischesWirtschaften werden unter der Creative-Commons-Lizenz "CC 4.0 Attribution Non-Commercial No Derivatives" veröffentlicht. Im Rahmen dieser Lizenz muss der Autor/Urheber stets genannt werden, das Werk darf nicht bearbeitet, abgewandelt oder in anderer Weise verändert und außerdem nicht kommerziell genutzt werden. Die digitale Version des Artikels bleibt für zwei Jahre Abonnent/innen vorbehalten und ist danach im Open Access verfügbar. 